genital tract (FGT), which are target cells for HIV infection. Recently, CD4 Th17 cells have been identified to be more susceptible to HIV infection. Here, we investigated the impact of the combined contraceptive vaginal ring (CCVR, NuvaRing), combined oral contraceptive pills (COCPs) and NET-EN on Th17-related cytokines in the FGT of adolescent girls.

Methods This was a randomized crossover trial with a duration of 8 months. Adolescent girls between the ages of 15-19 were recruited and assigned to NET-EN, CCVR and COCPs in a 1:1:1 ratio. After four months, participants crossed over to another product for an additional four months. Cervical supernatants were collected at baseline, crossover and exit visits. Fifteen Th17-related cytokines were measured using Luminex multiplex assays.

Results A total of 130 participants were enrolled at baseline, with 107 reaching visit 2 (crossover) and 92 completing the final visit. Baseline characteristics were similar across arms. Median concentrations of Th17-related cytokine did not differ at baseline across all arms. In an intention to treat analysis (ITT) at crossover, intraindividual analysis of participants on CCVR showed an increase in IL-21 ( $\mathrm{p}=0.009)$, IL-1 $1 \mathrm{p}=$ $0.007)$, TNF- $\alpha(p=0.01)$ and IFN- $\gamma(p=0.016)$. We did not see any intraindividual differences within the NET-EN and COCPs arm. Comparison across arms at both crossover and exit showed elevated Th17-related cytokines (including IL17A, IL-6, IL-1 $\beta$, IL-33, TNF- $\alpha$ ) in participants on CCVR compared to those on NET-EN and COCPs.

Conclusion In summary, the use of CCVR was associated with an increase in Th17-related cytokines compared to NET-EN and COCPs. Further studies are needed to investigate how these immune alterations in the FGT contribute to HIV risk in adolescent girls.

Disclosure No significant relationships.

\section{O05.4 THE EFFECT OF THE COMBINED ORAL CONTRACEPTIVE PILL ON THE VAGINAL MICROBIOTA OF WOMEN TREATED FOR BACTERIAL VAGINOSIS}

${ }^{1}$ Larissa Ratten* ${ }^{2}$ Catriona Bradshaw, ${ }^{2}$ Erica Plumer, ${ }^{2}$ Christopher Fairley, ${ }^{3}$ Gerald Murray, ${ }^{3}$ Suzanne Garland, ${ }^{3}$ Jennifer Danielewski, ${ }^{4}$ Gilda Tachedjian, ${ }^{2}$ Eric Chow, ${ }^{2}$ Lenka Vodstrcil. ${ }^{1}$ Monash University, Central Clinical School, Clayton, Australia; ${ }^{2}$ Monash University, Central Clinical School, Carlton, Australia; ${ }^{3}$ The Royal Women's Hospital, Centre for Women's Infectious Disease Research, Parkville, Australia; ${ }^{4}$ The Burnet Institute, Melbourne, Australia

\subsection{6/sextrans-2019-sti.131}

Background Bacterial Vaginosis (BV) is considered to be a dysbiosis of the vaginal microbiota (VM); it causes vaginal symptoms, increases risk for STI/HIV acquisition and negatively impacts obstetric outcomes. We analysed the VM of women from an open-label trial of women randomized to the combined-oral contraceptive pill (COCP) or current non-hormonal contraceptive practices after antibiotic treatment for BV. Our aim was to determine if COCP-exposure was associated with an optimal VM dominated by Lactobacillus spp. following antibiotic treatment.

Methods Women $(\mathrm{N}=92)$ returned vaginal swabs and questionnaires monthly for 6 months or until BV recurrence. Specimens $(\mathrm{N}=449)$ underwent $\mathrm{VM}$ analysis by $16 \mathrm{~S}$ rRNA gene V3V4 amplicon sequencing. Alpha diversity was calculated using the Shannon diversity index. Associations between behavioural factors and diversity were investigated using generalized estimating equations population-averaged models and multinomial regression was used to assess factors associated with composition.

Results Specimens were grouped into five VM types: Lactobacillus iners dominated, L. crispatus dominated, mixed Lactobacillus spp., Gardnerella vaginalis dominated and mixed highly diverse taxa. COCP-exposure was associated with a decrease in VM diversity (Shannon; adjusted coefficient $=-0.55$, 95\% CI:-0.75, 0.36,p<0.001). Women with COCP-exposure were also more likely to have VM dominated by either $L$. iners (adjusted relative risk ratio $[\mathrm{RRR}]=4.40,95 \% \mathrm{CI}$ : $1.90,10.18$, $\mathrm{p}=0.001$ ) or $L$. crispatus (adjRRR $=3.12$, 95\%CI:1.24,7.81, $\mathrm{p}=0.015$ ) than one dominated by $G$. vaginalis. Conversely, women who reported an ongoing regular sex partner (RSP) were more likely to have a VM dominated by $G$. vaginalis (adjRRR $=2.56,95 \% \mathrm{CI}: 0.80,8.22, \mathrm{p}=0.144)$ or mixed diverse taxa (adjRRR $=2.01,95 \% \mathrm{CI}: 0.81,4.99, \mathrm{p}=0.129)$ than by $L$. crispatus, although this was not significant.

Conclusion COCP-exposure is associated with higher relative abundance of Lactobacillus spp. and an increased likelihood of developing a VM dominated by L. crispatus or L. iners following antibiotic treatment. Conversely, re-exposure to an RSP increased the likelihood of a VM that was abundant in BVassociated bacteria including $G$. vaginalis. These findings have important implications for the development of BV treatment and prevention strategies.

Disclosure No significant relationships.

\subsection{A CASE CONTROL STUDY TO EXAMINE THE CERVICO- VAGINAL MICROBIOTA ASSOCIATED WITH PELVIC INFLAMMATORY DISEASE}

${ }^{1}$ Wilhelmina Huston, ${ }^{1}$ Rami Mazraani, ${ }^{1}$ Catherine Burke, ${ }^{2}$ Jacques Ravel, ${ }^{3}$ Kirsteen Flemming, ${ }^{3}$ Sally Sweeney*, ${ }^{3}$ Deborah Bateson. ${ }^{1}$ The University of Technology Sydney, School of Life Sciences, Ultimo, Australia; ${ }^{2}$ University of Maryland, Institute for Genome Sciences, Washington, USA; ${ }^{3}$ Family Planning New South Wales, Ashfield, Australia

\subsection{6/sextrans-2019-sti. 132}

Background This is a case-control study comparing women presenting with pelvic inflammatory disease (PID) (cases), and women presenting for routine cervical and/or sexually transmitted infection screening (cases) to examine the cervico-vaginal microbiota associated with PID. Currently, there is limited understanding of the association of the cervico-vaginal microbiota with PID.

Methods The study design is a case-control study with prospective recruitment of women presenting with PID and asymptomatic women presenting for routine cervical or sexual health screening. Cervical and posterior vaginal fornix specimens are collected for the study for microbiota (presented here) and gene expression analysis. Participant demographic data, clinical chart review to ensure consistency of recruitment and response to treatment of PID cases, and self-collected questionnaire on sexual, reproductive, and gynaecological history were also analysed. Antibiotic treatment in the month preceding recruitment and pregnancy were exclusion criteria. Results The study is still progressing, to date 38 control participants have been recruited and 12 cases consistent with PID. The analysis indicates that Lactobacillus iners (referred to as community state type 3 in vaginal microbiome) dominant vaginal microbial communities were significantly more frequently detected in cases. Additionally, cases were significantly more likely to have taken antibiotics in the past year, had 
recent partner change and/or self reported vaginal symptoms such as thrush or BV in the preceding 3 months. These are all factors which would potentially impact vaginal microbiota. Conclusion Pelvic inflammatory disease is known to be associated with sexual behaviour but a sexually transmitted infection is not always detected in PID. Here, we have found preliminary indications that a microbiota previously associated with sexually transmitted infection risk is also associated with PID. This is pilot data and further numbers are needed before conclusions can be reached.

Disclosure No significant relationships.

\subsection{CERVICOVAGINAL METABOLIC PROFILING REVEALS THE INTERPLAY BETWEEN HPV, MICROBIOTA AND INFLAMMATION IN CERVICAL CARCINOGENESIS}

'Zehra Ilhan, ${ }^{2}$ Pawel Laniewski, ${ }^{2}$ Natalie Thomas, ${ }^{3}$ Denise Roe, ${ }^{1}$ Dana Chase, ${ }^{4}$ Melissa Herbst-Kralovetz*. 'University of Arizona, Obstetrics and Gynecology, Phoenix, USA; ${ }^{2}$ University of Arizona, Basic Medical Sciences, Phoenix, USA; ${ }^{3}$ University of Arizona, Cancer Center, Tucson, USA; ${ }^{4}$ University of Arizona, Basic Medical Sciences/obstetrics and Gynecology, Phoenix, USA

\subsection{6/sextrans-2019-sti.133}

Background Vaginal dysbiosis has emerged as a key risk factor in HPV acquisition, persistence, and potentially cervical carcinogenesis. However, the biological mechanisms driving persistence and carcinogenesis have not been elucidated. Hence, our objective was to perform metabolic profiling of the cervicovaginal microenvironment to identify interactions between virus, host and microbes in the context of genital inflammation, dysplasia, and cancer.

Methods In a multicenter study, metabolic profiles of 78 premenopausal, non-pregnant women with low-grade (LSIL) and high-grade squamous intraepithelial lesions (HSIL), invasive cervical cancer (ICC), or healthy controls (HPV-positive and negative Ctrl) were analyzed using gas chromatography-mass spectrometry. Metabolome and vaginal microbiome datasets were integrated using state-of-the-art bioinformatic tools (PICRUSt, AMON, and MIMOSA). Hierarchical clustering analysis (HCA) and principal component analysis (PCA) were employed to reveal the influence of genital inflammation, patient groups, and microbiota on metabolic profiles. Receiver Operating Characteristics (ROC) analysis was used to discriminate metabolites for each patient group. Statistical differences were tested using ANOVA or Mann-Whitney U test.

Results Metabolomes of ICC patients ( $\mathrm{n}=468$ metabolites) formed a distinct cluster on PCA and HCA plots, due to enrichment of membrane lipids. Amino acid and nucleotide metabolites were depleted in HPV-positive Ctrl, LSIL and HSIL groups $(\mathrm{P}<0.05)$. Microbial communities were predicted to alter amino acid and nucleotide metabolisms. Eicosenoate, 3-hydroxybutyrate, and oleate/vaccenate (AUC >0.9, $\mathrm{P}<0.01$ ) discriminated ICC from healthy patients. Sphingolipids and plasmalogens positively correlated with genital inflammation (Spearman's rho > 0.7). Anti-inflammatory nucleotides, adenosine and cytosine positively correlated with Lactobacillus abundance (Spearman's rho>0.5) and negatively correlated with genital inflammation (Spearman's rho $<-0.3$ ). HCA of metabolites demonstrated that metabolic profiles were driven by cancer, genital inflammation and Lactobacillus dominance.
Conclusion The complex virus-host-microbe interplay within the cervicovaginal microenvironment lead to unique metabolic fingerprints that could be exploited for future development of diagnostics, preventatives or treatments to positively impact women's health outcomes.

Disclosure No significant relationships.

\section{6 - STI/HIV TREATMENT EFFICACY AND EFFECTIVENESS}

Monday, July 15, 2019 4:15 PM - 5:45 PM

\subsection{GENTAMICIN FOR PHARYNGEAL GONORRHEA: A SINGLE-ARM, NON-BLINDED CLINICAL TRIAL}

${ }^{1}$ Lindley Barbee*, ${ }^{2}$ Olusegun Soge, ${ }^{3}$ Jennifer Morgan, ${ }^{1}$ Angela Leclair, ${ }^{1}$ Tamara Bass, ${ }^{4}$ James Hughes, ${ }^{1}$ Matthew Golden. 'University of Washington, Medicine, Seattle, USA; ${ }^{2}$ University of Washington, Global Health and Medicine (infectious Diseases), Seattle, USA; ${ }^{3}$ Public Health - Seattle and King County, HIVISTD Program, Seattle, USA; ${ }^{4}$ University of Washington, Biostatistics, Seattle, USA

10.1136/sextrans-2019-sti.134

Background CDC guidelines recommend gentamicin for the treatment of gonorrhea in cephalosporin-allergic patients. The efficacy of gentamicin in the treatment of pharyngeal gonorrhea, which is often undiagnosed, is uncertain.

Methods Between September 2018 - March 2019, we enrolled MSM with NAAT-diagnosed pharyngeal gonorrhea in a singlearm, unblinded clinical trial. Men received a single $360 \mathrm{mg}$ intramuscular (IM) dose of gentamicin at enrollment and underwent test-of-cure (TOC) by culture 4-7 days later. The study measured creatinine at enrollment and TOC, serum gentamicin concentration post-dose to establish peak concentration (Cmax), and standard antimicrobial minimal inhibitory concentrations (MIC). The trial was designed to establish a point estimate for the efficacy of gentamicin for pharyngeal gonorrhea. We planned to enroll 50 evaluable subjects; assuming that gentamicin was $80 \%$ efficacious, the trial would establish a $95 \%$ confidence interval of $66 \%-90 \%$. We planned interim analyses at $n=10$ and $n=25$.

Results The study was stopped early due to poor efficacy. Of 13 enrolled men, 10 were evaluable, and only two $(20 \%$, 95\%CI: $2.5 \%-55.6 \%$ ) were cured. Of 2 concomitant rectal infections, both were cured. Efficacy was not associated with gentamicin Cmax $(p=0.809)$ or MIC $(p=0.429)$. No participants experienced renal insufficiency; average creatinine percent change was $5 \%$ (range: $-7 \%, 21 \%$ ). Six (46\%) subjects experienced headache; all deemed unrelated to treatment. On a scale of 0-10, mean injection pain was 2 (range: 1-7). Among subjects with history of bicillin $(n=7)$ and/or ceftriaxone $(n=8)$ IM injections, $86 \%$ believed IM gentamicin to be less painful than bicillin and $75 \%$ believed it was more painful than ceftriaxone.

Conclusion $360 \mathrm{mg}$ of gentamicin failed to eradicate N. gonorrhoeae from the pharynx. Caution should be used when using the CDC's current alternative therapy (gentamicin $240 \mathrm{mg}$ plus azithromycin 2g) given increases in azithromycin resistance and gentamicin's poor efficacy at the pharynx.

Disclosure No significant relationships. 